\title{
火烧信号对种子萌发影响的研究进展
}

\author{
李绍阳 ${ }^{1,2}$ 马红媛 ${ }^{*}$ 赵丹丹 1,3 马梦谣 ${ }^{1}$ 元雯雯 ${ }^{1}$ \\ ${ }^{1}$ 中国科学院东北地理与农业生态研究所, 长春 $130102 ;{ }^{2}$ 中国科学院大学, 北京 $100049 ;{ }^{3}$ 滨州学院, 山东省黄河三角洲生态环境重点实验室, 山东滨 \\ 州 256603
}

摘 要 火作为一个基础的生态因子, 对森林、草地等陆地生态系统的结构和功能有着重要的影响。种子萌发是种子植物的 重要生活史阶段, 也是火后植被更新和恢复的主要途径。植被燃烧产生了烟、热以及与烟相关的一系列火烧信号, 在打破种 子休眠, 促进种子萌发方面发挥重要作用。该文将火烧信号分为物理信号和化学信号, 物理信号主要是伴随火烧产生的高温, 化学信号主要包括气态烟以及近年来从烟水中提取的影响种子萌发的关键化学物质karrikins和glyceronitrile。该文围绕火烧的 基本信息，火烧信号对种子萌发的影响，火烧信号在实践中的应用 3 个方面进行系统综述，重点探讨了不同类别的火烧信号 及其交互作用对种子萌发的影响。在系统总结火烧信号对种子萌发影响的研究进展的基础上，提出未来的研究应与烟信号作 用机理的探究以及全球变化等方面相结合, 旨在充分发挥火的生态服务功能, 为火的科学管理应用和退化生态系统恢复提供 理论支撑。

关键词 种子萌发; 火烧; 物理信号; 化学信号; 交互作用

李绍阳, 马红媛, 赵丹丹, 马梦谣, 元雯雯 (2021). 火烧信号对种子萌发影响的研究进展. 植物生态学报, 45, 1177-1190. DOI: 10.17521/cjpe.2021.0123

\section{Research progress on the effects of fire-related cues on seed germination}

LI Shao-Yang ${ }^{1,2}$, MA Hong-Yuan ${ }^{1 *}$, ZHAO Dan-Dan $^{1,3}$, MA Meng-Yao ${ }^{1}$, and QI Wen-Wen ${ }^{1}$

${ }^{1}$ Northeast Institute of Geography and Agroecology, Chinese Academy of Sciences, Changchun 130102, China; ${ }^{2}$ University of Chinese Academy of Sciences, Beijing 100049, China; and ${ }^{3}$ Shandong Key Laboratory of Eco-Environmental Science for Yellow River Delta, Binzhou University, Binzhou, Shandong 256603, China

\begin{abstract}
Fire is a fundamental ecological factor profoundly affecting many terrestrial ecosystems including forest and grassland ecosystems. Seed germination is an important life-history stage of plants, and it is also the main strategy for the regeneration and restoration of vegetation after fire events. Vegetation combustion produces smoke, heat and a series of fire cues associated with smoke, and plays critical roles in breaking seed dormancy and seedling establishment. In this study, we clustered the fire cues into two categories, i.e., physical cues (heat) and chemical cues (aerial smoke, karrikins, glyceronitrile) and focused on the basic information of fire, the effects of fire cues on seed germination, and the application of fire cues. In addition, we focused on the effects of different types of fire cues and their interactions on seed germination. Based on a systematic review of the research progress on the effects of fire cues on seed germination, this study also provided suggestions for the future research, such as mechanism of smoke cues on seed germination and seedling growth via molecular technologies, and interaction of smoke cues with climate changes. In summary, based on the review of the ecological functions of fire-related cues on seed germination, this study aims to provide theoretical support for the scientific management of grassland and forest ecosystems and also provide basis for the applications of fire and the restoration of degraded ecosystems.
\end{abstract}

Key words seed germination; fire; physical cues; chemical cues; interaction

Li SY, Ma HY, Zhao DD, Ma MY, Qi WW (2021). Research progress on the effects of fire-related cues on seed germination. Chinese Journal of Plant Ecology, 45, 1177-1190. DOI: 10.17521/cjpe.2021.0123

火烧作为一个基础的生态过程, 深刻影响着森 林、草原等陆地生态系统(Bond et al., 2005; 王谢等, 2013; 杨健等, 2013; Pausas \& Keeley, 2019; McLau- chlan et al., 2020)。火在参与塑造全球生物群系的分 布，维持群落结构和功能的同时，也作为一种自然 选择力量, 驱动了物种的进化(Bond \& Keeley, 2005;

收稿日期Received: 2021-04-06 接受日期Accepted: 2021-09-07

基金项目: 国家自然科学基金(41977424和41771058)、中国科学院A类战略性先导科技专项(XDA2306040303和XDA28110300)和吉林省自然科学基 金(20200201026JC)。Supported by the National Natural Science Foundation of China (41977424 and 41771058), the Category A Strategic Priority Research Program of Chinese Academy of Sciences (XDA2306040303 and XDA28110300), and the Natural Science Foundation of Jilin Province (20200201026JC).

* 通信作者Corresponding author (mahongyuan@iga.ac.cn) 
He et al., 2011; Keeley et al., 2011)。

火烧可以影响植物的开花、种子传播、萌发、 幼苗建植和死亡等生长发育的各个方面(Todorović et al., 2010)。种子萌发是火烧后植被更新和恢复的 主要方式。土壤种子库及植冠种子库作为退化植被 动态变化的重要组成部分, 直接影响着生态系统的 恢复能力(Qiu et al., 2010; Lü et al., 2017)。火烧可以 降低物种间的竞争和提高资源的可利用性，从而为 种子萌发和幼苗建植提供有利环境, 进而实现种群 的延续(Purdie, 1977; Kemp et al., 2019)。植被燃烧产 生了烟、热、karrikins (KARs)、glyceronitrile $\left(\mathrm{SP}_{1}\right)$ 等火烧信号。这些信号在打破种子休眠, 促进种子 萌发方面发挥了重要作用。例如, 火烧显著增加了 Molinia caerulea 的生物量、结实率和萌发率(Brys et al., 2005)。Cuello等(2020)探究了乌拉圭温带草原土 壤种子库对于火烧的响应情况, 结果表明火烧处理 显著增加了种子萌发的密度和丰富度, 提前了萌发 高峰。火烧信号可以促进澳大利亚西南地区将近900 种植物种子的萌发(Auld \& Denham, 2006)。火烧信 号对种子萌发的影响在农业、园艺生产, 提高种子 抗逆性和植被恢复等方面具有广泛的应用(He et al., 2019; Pausas \& Keeley, 2019)。

本文分别从火烧信号的基本信息, 火烧信号对 种子萌发的影响, 火烧信号在实践中的应用 3 个方 面进行系统地综述, 重点探讨了不同类别的火烧信 号及其交互作用对种子萌发的影响, 并对未来的重 点研究方向进行了展望, 旨在充分发挥火的生态服 务功能, 为火的科学管理应用和退化生态系统恢复 提供理论支撑。

\section{1 火烧概况}

\section{1 火在陆地生态系统中的发生}

陆地生态系统中约 $90 \%$ 的火烧事件是由人类活 动导致, $10 \%$ 是由闪电和火山爆发等自然原因引起 (Shikwambana \& Kganyago, 2021)。火烧主要发生在 地中海沿岸、美国加利福尼亚沿岸、澳大利亚西南 部等地中海气候区(Moreira \& Pausas, 2018; StevensRumann \& Morgan, 2019)。温带干旱半干旱气候区、 热带干旱半干旱气候区的森林和草原也属于易发生 火灾的生态系统(Hodges et al., 2019; Ramos et al., 2019)。丰富的可燃物储量, 干热的天气和气候为这 些地区的火灾频发创造了条件(Bowman et al.,
2009)。在易发生火烧的生态系统中，植物已经进化 出一些适应性特征用于保证物种在火烧环境中存活 和火烧后植被的恢复和更新(Keeley et al., 2011)。在 不易发生火烧的生态系统中, 系统内部缺乏足够的 燃料和合适的气候条件来支持火烧现象的发生。然 而全球气候和土地利用方式的变化改变了火烧发生 地区的气候、地形、燃料等条件，加剧了火烧情势 的时空变化(Morgan et al., 2001; 吕爱锋和田汉勤, 2007; Kelly et al., 2020; 岳超等, 2020)。Silveira等 (2020)探究了2019年巴西热带雨林大火发生的原因, 结果表明森林砍伐程度高的地区其月度火烧发生的 频率显著高于砍伐程度低的地区。

\section{2 火烧信号}

根据火烧产物的理化性质将其分为物理和化学 信号两个类别(图1)。植物燃烧产生的含氮化合物、 烟、KARs和 $\mathrm{SP}_{1}$ 等萌发刺激物质被称为化学信号。 燃烧过程中产生的高温和燃烧后光照条件的变化属 于物理信号。火烧对于土壤的增温效果受燃料的生 物量、组成成分和含水量, 土壤的湿度、深度等因 素影响会呈现时空上的差异(Liyanage \& Ooi, 2015; Veenendaal et al., 2018)。表层土壤(0-3 cm) 是土壤种 子库集中分布的区域，火烧可以使该区域的温度增 至60-120 ${ }^{\circ} \mathrm{C}$ (Bradstock \& Auld, 1995)。然而不同的 生态系统如森林、草地等, 其火烧情势差异很大, 需 要深入调查不同生态系统的热激温度和持续时间, 从而更好地模拟热激这一物理信号对种子萌发的影 响。此外, 火烧对地表植被的破坏改变了到达地表 的光强和光质, 对需光或者嫌光种子的萌发具有重 要影响(López-Mársico et al., 2019)。

火烧后, 植物源烟中促进种子萌发的化学物质 会沉积在土壤表面。这些萌发刺激物质具有挥发性 和水溶性(Staden et al., 2000)。萌发刺激物质溶于水 形成烟水, 既可以避免挥发, 实现长久保存, 又可 以从地表转移到地下, 透过种皮, 刺激土壤种子库 中的种子萌发(Brown \& van Staden, 1997)。因此火 烧后一场降雨对于火烧生境中的植被恢复和种子萌 发具有重要意义(Quintana et al., 2004)。在实验室中, 将植物燃烧产生的烟雾经抽气装置抽取通过去离子 水制得实验所需的烟水(Coons et al., 2014)。烟水在 制备过程中的水量和燃料量决定了烟水的浓度。此 外, 火烧后产生的灰烬中含有大量的无机离子如 $\mathrm{K}^{+} 、 \mathrm{NO}_{3}^{-} 、 \mathrm{NO}_{2}^{-}$等，同样会影响火烧生境下种子的 


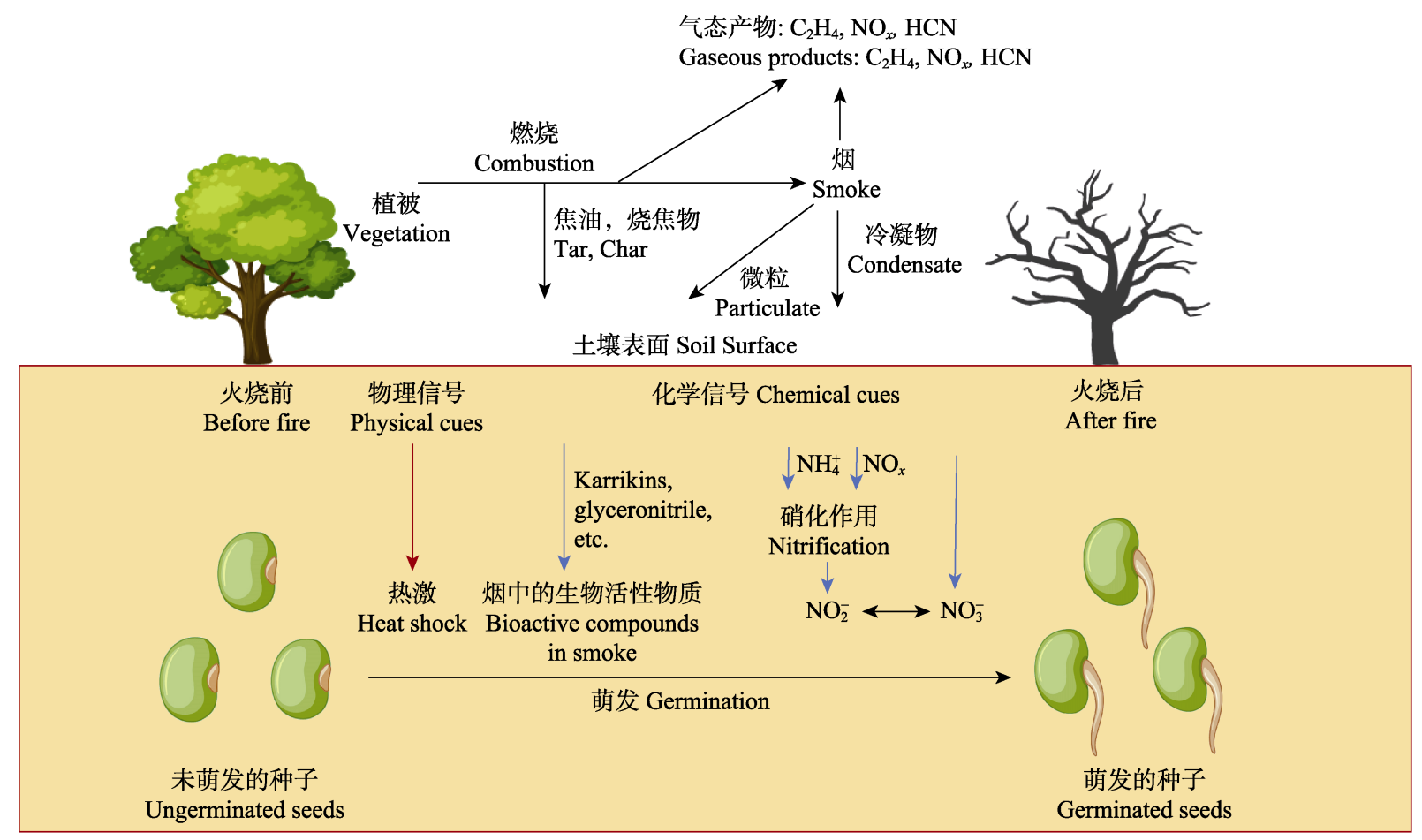

图1 影响种子萌发的火烧信号, 修改自Nelson等(2012)和Kamran等(2014)。红色和蓝色箭头分别表示植被燃烧产生的物理和 化学信号。两种信号单独或者交互作用于种子萌发。热激的温度会随着土壤深度的增加而降低。化学信号可以借助雨水进入 到土壤中。 $\mathrm{NO}_{x}$ 代表 $\mathrm{NO}$ 或者 $\mathrm{NO}_{2}$, 通过硝化作用, $\mathrm{NO}_{x}$ 或者 $\mathrm{NO}_{4}^{+}$氧化变成 $\mathrm{NO}_{2}^{-}$或者 $\mathrm{NO}_{3}^{-}$。

Fig. 1 Fire cues of affecting seed germination, modified from Nelson et al. (2012) and Kamran et al. (2014). The red and blue arrows represent the physical and chemical cues produced by vegetation combustion, respectively. Two cues individually or interactively affect seed germination. The heat shock temperature decreases with increasing soil depth. Chemical cues would normally be eluted into the soil by rain. $\mathrm{NO}_{x}$ represents $\mathrm{NO}$ or $\mathrm{NO}_{2}$. Oxidation of $\mathrm{NH}_{4}^{+}$or $\mathrm{NO}_{x}$ to $\mathrm{NO}_{2}^{-}$or $\mathrm{NO}_{3}^{-}$can occur by nitrification.

萌发(Downes et al., 2014; Mackenzie \& Naeth, 2019)。但是这些物质并非是火烧所特有的产物，当 前关于化学信号对种子萌发影响的研究多集中在烟 信号以及从中提取的萌发刺激物质等方面。

KARs 是由植被燃烧产生的存在于烟和烟水中 一系列小的丁烯酸内酯分子的总称(Yao \& Waters, 2020)。植物源烟中成分复杂, 含有上千种化合物, Flematti等(2004)将烟水中化合物进行分离、测定, 最终发现了对种子萌发具有刺激作用的生物活性化 学物质karrikinolide $\left(\mathrm{KAR}_{1}\right)$ (图2A)。目前已经从植物 源烟中发现了6种 $\mathrm{KAR}\left(\mathrm{KAR}_{1-6}\right)$, 其中 $\mathrm{KAR}_{1-4}$ 比较 活跃(Nelson et al., 2012)。KARs在紫外线下不稳定, 自然光可导致其含量减少(Scaffidi et al., 2012)。 KARs 是烟中的主要萌发刺激物质, 但以Anigozanthos manglesii为代表的部分植物其萌发对烟水响 应而对KARs不响应, 促使了新的萌发刺激化合物 glyceronitrile的发现(图2B)。Glyceronitrile是氰醇类物 质，可以通过缓慢地释放氧化物刺激部分植物种子 萌发(Flematti et al., 2011)。Preston和Baldwin (1999)<smiles>COc1ccc2oc(=O)c(C)c-2o1</smiles>

Karrikinolide<smiles>N#CC(O)CO</smiles>

Glyceronitrile
图2 植物源烟中关键生物活性物质的化学结构。参考自 Flematti等(2011)。

Fig. 2 Chemical structure of key bioactive compounds in plant-derived smoke. Redrawed from Flematti et al. (2011).

研究发现火烧发生后萌发刺激物质可以在土壤中存 在超过7年时间, 对种子萌发的影响具有持久性。

\section{2 火烧信号及其互作对于种子萌发的影响}

在陆地生态系统中诸多一年生草本、多年生草 本、灌木和乔木等不同生活史特征的植物种子萌发 皆会对火烧信号产生响应(Nelson et al., 2012; Ferraz et al., 2013; Ramos et al., 2019)。目前对影响种子萌 发的火烧信号的研究主要集中在物理信号(热激效 应), 化学信号(烟、烟水、KARs、 $\mathrm{SP}_{1}$ ), 以及物理和 化学信号的交互作用。 


\section{1 物理信号}

热激效应刺激种子萌发的现象已经在豆科、菊 科、半日花科等多种植物类群中得到验证(表1)。热 激通过破坏种皮/果皮厚实的不透水层，打破种子 的物理休眠，促进种子萌发(Baskin et al., 2000)。物 理休眠是控制种子库动态变化的主导机制, 持久种
子库对于火烧后植被恢复具有重要意义(Baskin \& Baskin, 2014; Ooi et al., 2014)。Auld和O'connell (1991)发现，热激促进了澳大利亚东部地区35种豆 科植物种子的萌发, 并且可以根据物种间打破休眠 所需温度阈值 $\left(40 、 60 、 80{ }^{\circ} \mathrm{C}\right)$ 的差异，将其分为不 同的类别。Moreira等(2010)探究了地中海沿岸豆科、

表1 热激效应对于种子萌发的影响

Table 1 Effects of heat shock on seed germination

\begin{tabular}{|c|c|c|}
\hline 科 Family & 物种 Species & 实验设计 Experimen \\
\hline 豆科 & 鹰爪豆 & $T\left(80,110,150{ }^{\circ} \mathrm{C}\right)$ \\
\hline Fabaceae & Spartium junceum & $t(5,10 \mathrm{~min})$ \\
\hline $\begin{array}{l}\text { 豆科 } \\
\text { Fabaceae }\end{array}$ & Bituminaria bituminosa & $\begin{array}{l}T\left(80,110,150{ }^{\circ} \mathrm{C}\right) \\
t(5,10 \mathrm{~min})\end{array}$ \\
\hline
\end{tabular}

$\begin{array}{lll}\text { 豆科 } & \text { 阿拉伯胶树 } & T\left(60,90,120,150{ }^{\circ} \mathrm{C}\right) \times \\ \text { Fabaceae } & \text { Senegalia senegal } & t(1,5,10 \mathrm{~min})\end{array}$

$\begin{array}{lll}\text { 豆科 } & \text { 金雀儿 } & T\left(80,110,150{ }^{\circ} \mathrm{C}\right) \times \\ \text { Fabaceae } & \text { Cytisus scoparius } & t(5,10 \mathrm{~min})\end{array}$

\begin{tabular}{|c|c|c|}
\hline $\begin{array}{l}\text { 豆科 } \\
\text { Fabaceae }\end{array}$ & Genista tridentata & $\begin{array}{l}T\left(80,110,150{ }^{\circ} \mathrm{C}\right) \times \\
t(5,10 \mathrm{~min})\end{array}$ \\
\hline $\begin{array}{l}\text { 豆科 } \\
\text { Fabaceae }\end{array}$ & Cytisus striatus & $\begin{array}{l}T\left(80,110,150{ }^{\circ} \mathrm{C}\right) \times \\
t(5,10 \mathrm{~min})\end{array}$ \\
\hline $\begin{array}{l}\text { 豆科 } \\
\text { Fabaceae }\end{array}$ & Genista triacanthos & $\begin{array}{l}T\left(80,100,120{ }^{\circ} \mathrm{C}\right) \times \\
t(5,10 \mathrm{~min})\end{array}$ \\
\hline $\begin{array}{l}\text { 豆科 } \\
\text { Fabaceae }\end{array}$ & Viminaria juncea & $\begin{array}{l}T(40,60,80,100,120 \\
t(10 \mathrm{~min})\end{array}$ \\
\hline
\end{tabular}

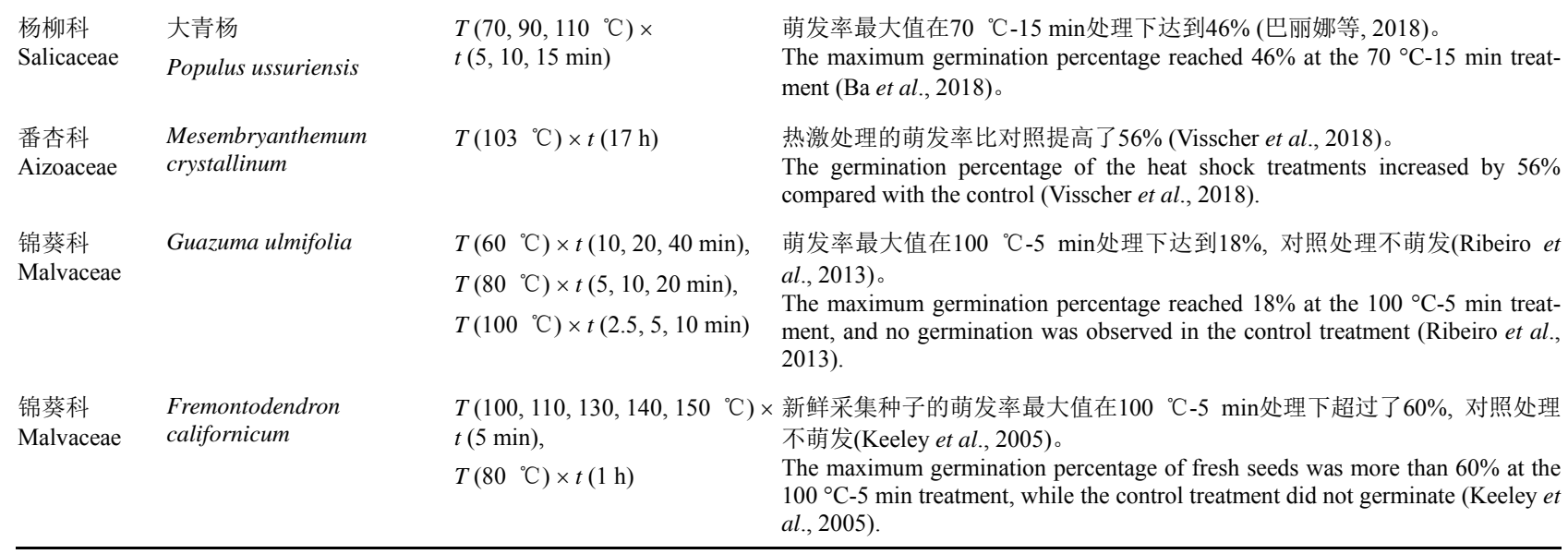


表 1 (续) Table 1 (Continued)

\begin{tabular}{|c|c|c|c|}
\hline 科 Family & 物种 Species & 实验设计 Experiment design & 主要发现 Main findings \\
\hline $\begin{array}{l}\text { 半日花科 } \\
\text { Cistaceae }\end{array}$ & Cistus albidus & $\begin{array}{l}T\left(80,100,120{ }^{\circ} \mathrm{C}\right) \times \\
t(5,10 \mathrm{~min})\end{array}$ & $\begin{array}{l}\text { 萌发率最大值在 } 120{ }^{\circ} \mathrm{C}-5 \mathrm{~min} \text { 处理下达到 } 61 \% \text {, 同对照相比提高了 } 50 \% \\
\text { (Moreira et al., 2010)。 } \\
\text { The maximum germination percentage reached } 61 \% \text { at the } 120{ }^{\circ} \mathrm{C}-5 \text { min treat- } \\
\text { ment, which was } 50 \% \text { higher than the control (Moreira et al., 2010). }\end{array}$ \\
\hline $\begin{array}{l}\text { 半日花科 } \\
\text { Cistaceae }\end{array}$ & Cistus monspeliensis & $\begin{array}{l}T\left(80,100,120{ }^{\circ} \mathrm{C}\right) \times \\
t(5,10 \mathrm{~min})\end{array}$ & $\begin{array}{l}\text { 萌发率最大值在 } 120{ }^{\circ} \mathrm{C}-5 \mathrm{~min} \text { 处理下达到 } 73 \% \text {, 同对照相比提高了 } 62 \% \\
\text { (Moreira et al., 2010)。 } \\
\text { The maximum germination percentage reached } 73 \% \text { at the } 120{ }^{\circ} \mathrm{C}-5 \text { min treat- } \\
\text { ment, which was } 62 \% \text { higher than the control (Moreira et al., 2010). }\end{array}$ \\
\hline $\begin{array}{l}\text { 半日花科 } \\
\text { Cistaceae }\end{array}$ & Cistus creticus & $\begin{array}{l}T\left(80,100,120,150{ }^{\circ} \mathrm{C}\right) \times \\
t(5 \mathrm{~min})\end{array}$ & $\begin{array}{l}\text { 萌发率在100、120、150 }{ }^{\circ} \mathrm{C} \text { 处理下显著高于对照处理(Tavsanoglu, 2011)。 } \\
\text { The germination percentage at } 100,120 \text { and } 150^{\circ} \mathrm{C} \text { treatments was significantly } \\
\text { higher than the control (Tavsanoglu, 2011). }\end{array}$ \\
\hline $\begin{array}{l}\text { 半日花科 } \\
\text { Cistaceae }\end{array}$ & Helianthemum apenninum & $\begin{array}{l}T\left(80,100,120{ }^{\circ} \mathrm{C}\right) \times \\
t(10 \mathrm{~min})\end{array}$ & $\begin{array}{l}\text { 萌发率最大值在 } 80{ }^{\circ} \mathrm{C}-10 \mathrm{~min} \text { 处理下达到 } 39 \% \text {, 同对照相比提高了 } 19 \% \\
\text { (Luna et al., 2007)。 } \\
\text { The maximum germination percentage reached } 39 \% \text { at the } 80{ }^{\circ} \mathrm{C}-10 \text { min treat- } \\
\text { ment, which was } 19 \% \text { higher than the control (Luna et al., 2007). }\end{array}$ \\
\hline $\begin{array}{l}\text { 唇形科 } \\
\text { Labiatae }\end{array}$ & $\begin{array}{l}\text { 宽叶薰衣草 } \\
\text { Lavandula latifolia }\end{array}$ & $\begin{array}{l}T\left(80,100,120{ }^{\circ} \mathrm{C}\right) \times \\
t(5,10 \mathrm{~min})\end{array}$ & $\begin{array}{l}\text { 萌发率最大值在 } 100{ }^{\circ} \mathrm{C}-5 \mathrm{~min} \text { 处理下达到 } 32 \% \text {, 同对照相比提高了 } 19 \% \\
\text { (Moreira et al., 2010)。 } \\
\text { The maximum germination percentage reached } 32 \% \text { at the } 100{ }^{\circ} \mathrm{C}-5 \text { min treat- } \\
\text { ment, which was } 19 \% \text { higher than the control (Moreira et al., 2010). }\end{array}$ \\
\hline $\begin{array}{l}\text { 唇形科 } \\
\text { Labiatae }\end{array}$ & Lavandula stoechas & $\begin{array}{l}T\left(80,100,120{ }^{\circ} \mathrm{C}\right) \times \\
t(5,10 \mathrm{~min})\end{array}$ & $\begin{array}{l}\text { 萌发率最大值在 } 120{ }^{\circ} \mathrm{C}-5 \mathrm{~min} \text { 处理下达到 } 72 \% \text {, 同对照相比提高了 } 62 \% \\
\text { (Moreira et al., 2010)。 } \\
\text { The maximum germination percentage reached } 72 \% \text { at the } 120{ }^{\circ} \mathrm{C}-5 \text { min treat- } \\
\text { ment, which was } 62 \% \text { higher than the control (Moreira et al., 2010). }\end{array}$ \\
\hline $\begin{array}{l}\text { 菊科 } \\
\text { Asteraceae }\end{array}$ & Catananche caerulea & $\begin{array}{l}T\left(80,100,120^{\circ} \mathrm{C}\right) \times \\
t(10 \mathrm{~min})\end{array}$ & $\begin{array}{l}\text { 萌发率最大值在 } 80{ }^{\circ} \mathrm{C}-10 \mathrm{~min} \text { 处理下达到 } 55 \% \text {, 同对照相比提高了 } 44 \% \\
\text { (Luna et al., 2007)。 } \\
\text { The maximum germination percentage reached } 55 \% \text { at the } 80{ }^{\circ} \mathrm{C}-10 \text { min treat- } \\
\text { ment, which was } 44 \% \text { higher than the control (Luna et al., 2007). }\end{array}$ \\
\hline $\begin{array}{l}\text { 禾本科 } \\
\text { Poaceae }\end{array}$ & $\begin{array}{l}\text { 小穗臭草 } \\
\text { Melica ciliata }\end{array}$ & $\begin{array}{l}T\left(80,110,150{ }^{\circ} \mathrm{C}\right) \times \\
t(5,10 \mathrm{~min})\end{array}$ & $\begin{array}{l}\text { 萌发率在 } 110{ }^{\circ} \mathrm{C}-5 \text { min 处理下达到最大值, } 150{ }^{\circ} \mathrm{C}-10 \mathrm{~min} \text { 热激处理完全抑制 } \\
\text { 了萌发(Reyes \& Trabaud, 2009)。 } \\
\text { The germination percentage reached the maximum at the } 110{ }^{\circ} \mathrm{C}-5 \text { min treat- } \\
\text { ment, and the germination was completely inhibited at the } 150{ }^{\circ} \mathrm{C}-10 \text { min treat- } \\
\text { ment (Reyes \& Trabaud, 2009). }\end{array}$ \\
\hline
\end{tabular}

$T$, 温度; $t$, 时间。

$T$, temperature; $t$, time.

半日花科、唇形科等 7 个科共 30 个物种种子萌发对于 热激的响应情况, 结果表明有 21 个物种在热激下萌 发率显著增加。热激的温度和持续时间是探讨热信 号对于种子萌发影响的两个重要变量, 高温度长时 间的热激会使种子失去活性(Ooi et al., 2014)。例如, 小穗臭草(Melica ciliata)的萌发率在 $110{ }^{\circ} \mathrm{C}-5 \mathrm{~min}$ 处 理下达到最大值, 但在 $150{ }^{\circ} \mathrm{C}-10 \mathrm{~min}$ 热激处理下则 完全抑制了萌发(Reyes \& Trabaud, 2009)。

相近类群的植物种子萌发对于热激效应的响应 并不完全一致。同属于相思树属(Acacia)的两个物种 的种子萌发对于热激的响应存在差异。在 $90{ }^{\circ} \mathrm{C}$ 热激 时, 只有阿拉伯胶树(Senegalia senegal)的萌发率显 著增加(Herrero et al., 2019)。Liyanage和Ooi (2015) 研究了澳大利亚东南部具有物理休眠的 5 种灌木种 子打破休眠所需的温度阈值在种群内的变化规律, 结果表明同一种群内部, 不同个体之间打破休眠所 需的温度阈值存在显著差异, 有的个体在较低温度 热激时便可以萌发, 有的则需要高温热激。
并不是所有物理休眠的物种种子萌发都会对热 激响应，同样热激也不是物理休眠种子的专属信 号。Ma等(2020)对形态生理休眠的Anigozanthos flavidus种子进行了 $100{ }^{\circ} \mathrm{C}-30 \mathrm{~min}$ 的热激处理, 结 果显示 12 个种群中有 6 个种群的种子萌发率显著提 高。一些生理休眠的物种也可以对热激产生萌发响 应。无论是储存的还是新鲜采集的Drosophyllum lusitanicum种子，经 $80{ }^{\circ} \mathrm{C}$ 或者 $100{ }^{\circ} \mathrm{C}$ 热激后萌发率 显著增加(Cross et al., 2017)。澳大利亚西南部 Darwinia的 5 个物种的种子皆具有透水性, 经过热 激处理后，5个物种的萌发率均显著增加(Auld \& Ooi, 2009)。

光是某些植物种子萌发所必需的环境条件 (Moreira \& Pausas, 2018)。火烧通过移除地表植被改 变了到达地表的光强和光质, 进而对部分植物的种 子萌发产生影响。同大种子植物相比, 小种子植物 的萌发对于光照有着更强的依赖性。Milberg等 (2000)探究了 54种植物种子萌发对于光照的响应情 
况, 并且在分析过程中考虑了系统发育因素, 结果 表明种子萌发对于光照的需求与种子大小出现了共 同进化。Roeder等(2019)发现火烧后开阔的生境可 以为小种子植物虾子花 (Woodfordia fruticosa) 的萌 发提供必需的光照条件。植被冠层的存在使到达地 表的红光和远红光的比例维持在一个较低的水平, 抑制了种子萌发(López-Mársico et al., 2019)。Baeza 和Roy (2008)研究发现, 火烧通过破坏冠层的完整 性改变了红光和远红光的比例, 显著提高了豆科植 物Ulex parviflorus种子的萌发率。目前对光信号的 研究主要集中在单一光因子对种子萌发的影响, 而
光信号与其他火烧信号的交互作用对种子萌发的影 响研究较少(Tavşanoğlu et al., 2017; López-Mársico et al., 2019)。

\section{2 化学信号}

火烧产生的一系列烟信号刺激种子萌发的现象 已经在菊科、十字花科、煘尾科等多个植物类群得 到验证(表2)。van de Venter和Esterhuizen (1988)率先 指出高温不是火烧信号中唯一影响种子萌发的因 素, 种子萌发与多种火烧信号密切相关。Keeley和 Fotheringham (1998)发现植物源烟可以促进美国加 利福尼亚地区 11 个科中 25 种灌木种子的萌发, 而这

表2 烟信号对于种子萌发的影响

Table 2 Effects of smoke cues on seed germination

\begin{tabular}{|c|c|c|c|}
\hline 科 Family & 物种 Species & 实验设计 Experiment design & 主要发现 Main findings \\
\hline $\begin{array}{l}\text { 菊科 } \\
\text { Asteraceae }\end{array}$ & Gutierrezia sarothrae & $S W(1: 5,1: 10,1: 100)$ & $\begin{array}{l}\text { 萌发率最大值在 } 1: 5 \text { 浓度下达到 } 46 \% \text {, 同对照相比增加了 } 14 \% \text { (Chou et al., } \\
\text { 2012)。 } \\
\text { The maximum germination percentage reached } 46 \% \text { at the concentration of } 1: 5 \text {, } \\
\text { which was } 14 \% \text { higher than the control (Chou et al., 2012). }\end{array}$ \\
\hline $\begin{array}{l}\text { 豆科 } \\
\text { Fabaceae }\end{array}$ & Astragalus crassicarpus & $S W(1: 5,1: 10,1: 100)$ & $\begin{array}{l}\text { 萌发率最大值在 } 1: 100 \text { 浓度下达到 } 28 \% \text {, 同对照相比增加了 } 8 \% \text { (Chou et al., } \\
2012 \text { )。 } \\
\text { The maximum germination percentage reached } 28 \% \text { at the concentration of } \\
\text { 1:100, which was } 8 \% \text { higher than the control (Chou et al., 2012). }\end{array}$ \\
\hline $\begin{array}{l}\text { 芸香科 } \\
\text { Rutaceae }\end{array}$ & Boronia floribunda & $S W(1: 10,1: 100)$ & $\begin{array}{l}\text { 同对照相比, 所有个体的萌发率经烟水处理后均显著增加(Ma et al., 2018)。 } \\
\text { Compared with the control, the germination percentage of all individuals were } \\
\text { significantly increased after smoke water treatments (Ma et al., 2018). }\end{array}$ \\
\hline $\begin{array}{l}\text { 十字花科 } \\
\text { Brassicaceae }\end{array}$ & $\begin{array}{l}\text { 小果亚麻荠 } \\
\text { Camelina microcarpa }\end{array}$ & $S W(1: 2)$ & $\begin{array}{l}\text { 萌发率在烟水处理下比对照提高了2.24倍(Mojzes \& Kalapos, 2014)。 } \\
\text { The germination percentage of the smoke water treatment increased by } 2.24 \text { times } \\
\text { compared with the control (Mojzes \& Kalapos, 2014). }\end{array}$ \\
\hline $\begin{array}{l}\text { 十字花科 } \\
\text { Brassicaceae }\end{array}$ & $\begin{array}{l}\text { 荠 } \\
\text { Capsella bursa-pastoris }\end{array}$ & $S W(1: 2)$ & $\begin{array}{l}\text { 萌发率在烟水处理下比对照提高了 } 3.20 \text { 倍(Mojzes \& Kalapos, 2014)。 } \\
\text { The germination percentage of the smoke water treatment increased by } 3.20 \text { times } \\
\text { compared with the control (Mojzes \& Kalapos, 2014). }\end{array}$ \\
\hline $\begin{array}{l}\text { 十字花科 } \\
\text { Brassicaceae }\end{array}$ & $\begin{array}{l}\text { 播娘蒿 } \\
\text { Descurainia sophia }\end{array}$ & $S W(1: 2)$ & $\begin{array}{l}\text { 萌发率在烟水处理下比对照提高了 } 4.21 \text { 倍(Mojzes \& Kalapos, 2014)。 } \\
\text { The germination percentage of the smoke water treatment increased by } 4.21 \\
\text { compared with the control (Mojzes \& Kalapos, 2014). }\end{array}$ \\
\hline $\begin{array}{l}\text { 十字花科 } \\
\text { Brassicaceae }\end{array}$ & Brassica tournefortii & $\begin{array}{l}\mathrm{KAR}_{1}(0.67,6.70 \\
67.00 \mathrm{nmol} \cdot \mathrm{L}^{-1} ; 0.67 \\
\left.6.70 \mu \mathrm{mol} \cdot \mathrm{L}^{-1}\right)\end{array}$ & $\begin{array}{l}\text { 萌发率最大值在 } 6.70 \mu \mathrm{mol} \cdot \mathrm{L}^{-1} \text { 浓度梯度下达到 } 93 \% \text {, 比对照增加了 } 89 \% \text { (Ste- } \\
\text { vens et al., 2007)。 } \\
\text { The maximum germination percentage reached } 93 \% \text { at the concentration of } \\
6.70 \mu \mathrm{mol} \cdot \mathrm{L}^{-1} \text { which was } 89 \% \text { higher than the control (Stevens et al., 2007). }\end{array}$ \\
\hline $\begin{array}{l}\text { 十字花科 } \\
\text { Brassicaceae }\end{array}$ & $\begin{array}{l}\text { 野萝卜 } \\
\text { Raphanus raphanistrum }\end{array}$ & $\begin{array}{l}\mathrm{KAR}_{1}(0.67,6.70 \\
67.00 \mathrm{nmol} \cdot \mathrm{L}^{-1} ; 0.67 \\
\left.6.70 \mu \mathrm{mol} \cdot \mathrm{L}^{-1}\right)\end{array}$ & $\begin{array}{l}\text { 萌发率最大值在 } 6.70 \mu \mathrm{mol} \cdot \mathrm{L}^{-1} \text { 浓度梯度下达到 } 91 \% \text {, 比对照增加了 } 40 \% \text { (Ste- } \\
\text { vens et al., 2007)。 } \\
\text { The maximum germination percentage reached } 91 \% \text { at the concentration of } \\
6.70 \mu \mathrm{mol} \cdot \mathrm{L}^{-1} \text {, which was } 40 \% \text { higher than the control (Stevens et al., 2007). }\end{array}$ \\
\hline $\begin{array}{l}\text { 车前科 } \\
\text { Plantaginaceae }\end{array}$ & $\begin{array}{l}\text { 长叶车前 } \\
\text { Plantago lanceolata }\end{array}$ & $S W(1: 2)$ & $\begin{array}{l}\text { 同对照相比，经烟水处理后萌发率提高了 } 41 \% \text { (Mojzes \& Kalapos, 2014)。 } \\
\text { The germination percentage of the smoke water treatment increased by } 41 \% \\
\text { compared with the control (Mojzes \& Kalapos, 2014). }\end{array}$ \\
\hline $\begin{array}{l}\text { 马癿铃科 } \\
\text { Aristolochiaceae }\end{array}$ & $\begin{array}{l}\text { 马悓铃 } \\
\text { Aristolochia debilis }\end{array}$ & $S W(1: 10)$ & $\begin{array}{l}\text { 同对照相比, 经烟水处理后萌发率提高了 } 27 \% \text { (Zhou et al., 2014)。 } \\
\text { The germination percentage of the smoke water treatment increased by } 27 \% \\
\text { compared with the control (Zhou et al., 2014). }\end{array}$ \\
\hline $\begin{array}{l}\text { 葫芦科 } \\
\text { Cucurbitaceae }\end{array}$ & $\begin{array}{l}\text { 黄瓜 } \\
\text { Cucumis sativus }\end{array}$ & $S W(100 \%)$ & $\begin{array}{l}\text { 同对照相比，经烟水处理后萌发率提高 } 11 \% \text { (Elsadek \& Yousef, 2019)。 } \\
\text { The germination percentage of the smoke water treatment increased by } 11 \% \\
\text { compared with the control (Elsadek \& Yousef, 2019). }\end{array}$ \\
\hline $\begin{array}{l}\text { 煘尾科 } \\
\text { Iridaceae }\end{array}$ & Gladiolus hybridus & $S W(100 \%)$ & $\begin{array}{l}\text { 同对照相比，经烟水处理后萌发率提高了 } 10 \% \text { (Elsadek \& Yousef, 2019)。 } \\
\text { The germination percentage of the smoke water treatment increased by } 10 \% \\
\text { compared with the control (Elsadek \& Yousef, 2019). }\end{array}$ \\
\hline $\begin{array}{l}\text { 唇形科 } \\
\text { Lamiaceae }\end{array}$ & Lavandula stoechas & $S W(1: 1,1: 10,1: 100)$ & $\begin{array}{l}\text { 萌发率最大值在 } 1: 100 \text { 浓度下达到 } 95 \% \text {, 同对照相比提高了 } 26 \% \text { (Çatav et al., } \\
2014 \text { )。 } \\
\text { The maximum germination percentage reached } 95 \% \text { at the concentration of } \\
1: 100 \text {, which was } 26 \% \text { higher than the control (Çatav et al., 2014). }\end{array}$ \\
\hline
\end{tabular}


表 2 (续) Table 2 (Continued)

\begin{tabular}{|c|c|c|c|}
\hline 科 Family & 物种 Species & 实验设计 Experiment design & 主要发现 Main findings \\
\hline $\begin{array}{l}\text { 唇形科 } \\
\text { Lamiaceae }\end{array}$ & Origanum onites & $S W(1: 1,1: 10,1: 100)$ & $\begin{array}{l}\text { 萌发率最大值在 } 1: 100 \text { 浓度下达到 } 97 \% \text {, 同对照相比提高了 } 14 \% \text { (Çatav et al., } \\
2014 \text { )。 } \\
\text { The maximum germination percentage reached } 97 \% \text { at the concentration of } \\
1: 100 \text {, which was } 14 \% \text { higher than the control (Çatav et al., 2014). }\end{array}$ \\
\hline $\begin{array}{l}\text { 唇形科 } \\
\text { Lamiaceae }\end{array}$ & Satureja thymbra & $S W(1: 1,1: 10,1: 100)$ & $\begin{array}{l}\text { 萌发率最大值在 } 1: 1 \text { 浓度下达到 } 87 \% \text {, 同对照相比提高了 } 30 \% \text { (Çatav et al., } \\
\text { 2014)。 } \\
\text { The maximum germination percentage reached } 87 \% \text { at the concentration of } 1: 1 \text {, } \\
\text { which was } 30 \% \text { higher than the control (Çatav et al., 2014). }\end{array}$ \\
\hline 禾本科 & 野燕麦 & $S W(1: 10000,1: 1000,1: 100)$ & 萌发率最大值在 $1: 100$ 浓度下达到 $90 \%$ 。 $\mathrm{KAR}_{1}$ 在 $15-30{ }^{\circ} \mathrm{C}$ 范围内可显著提高 \\
\hline Poaceae & Avena fatua & $\begin{array}{l}T\left(15,20,25,30{ }^{\circ} \mathrm{C}\right) \times \mathrm{KAR}_{1} \\
\left(10^{-7}, 10^{-8}, 10^{-9}, 10^{-10} \mathrm{~mol} \cdot \mathrm{L}^{-1}\right)\end{array}$ & $\begin{array}{l}\text { 种子萌发率(Kępczyński et al., 2006, 2010)。 } \\
\text { The maximum germination percentage reached } 90 \% \text { at the concentration of } \\
1: 100 \text {. KAR }{ }_{1} \text { significantly increased seed germination in the range of } 15-30{ }^{\circ} \mathrm{C} \\
\text { (Kępczyński et al., 2006, 2010). }\end{array}$ \\
\hline $\begin{array}{l}\text { 蓄薇科 } \\
\text { Rosaceae }\end{array}$ & Adenostoma fasciculatum & $S W(1: 100,1: 500,1: 1000)$ & $\begin{array}{l}\text { 萌发率在 } 1: 500 \text { 浓度下超过 } 90 \% \text { ，显著高于对照(Keeley et al., 2005)。 } \\
\text { The germination percentage was over } 90 \% \text { at the concentration of 1:500, which } \\
\text { was significantly higher than the control (Keeley et al., 2005). }\end{array}$ \\
\hline $\begin{array}{l}\text { 紫草科 } \\
\text { Boraginaceae }\end{array}$ & Eriodictyon crassifolium & $S W(1: 100,1: 500,1: 1000)$ & $\begin{array}{l}\text { 萌发率在 } 1: 500 \text { 浓度下超过 } 80 \% \text {, 显著高于对照(Keeley et al., 2005)。 } \\
\text { The germination percentage was over } 80 \% \text { at the concentration of 1:500, which } \\
\text { was significantly higher than the control (Keeley et al., 2005). }\end{array}$ \\
\hline $\begin{array}{l}\text { 血皮草科 } \\
\text { Haemodoraceae }\end{array}$ & Anigozanthos flavidus & $\begin{array}{l}\mathrm{SP}_{1}(5.00,10.00,25.00,50.00 \\
75.00,100.00,200.00,300.00 \\
\left.400.00,500.00 \mu \mathrm{mol} \cdot \mathrm{L}^{-1}\right)\end{array}$ & $\begin{array}{l}\text { 萌发率最大值在 } 50.00 \text { 和 } 200.00 \mu \mathrm{mol} \cdot \mathrm{L}^{-1} \text { 下达到 } 95 \% \text {, 比对照增加了 } 67 \% \\
\text { (Downes et al., 2013)。 } \\
\text { The maximum germination percentage reached } 95 \% \text { at } 50.00 \text { and } 200.00 \mu \mathrm{mol} \cdot \mathrm{L}^{-1} \text {, } \\
\text { which was } 67 \% \text { higher than the control (Downes et al., 2013). }\end{array}$ \\
\hline
\end{tabular}

$\mathrm{KAR}_{1}$, karrikinolide; $\mathrm{SP}_{1}$, glyceronitrile; $S W$, 烟水; $T$, 温度。

$\mathrm{KAR}_{1}$, karrikinolide; $\mathrm{SP}_{1}$, glyceronitrile; $S W$, smoke water; $T$, temperature.

些物种均不会对热信号响应。Çatav等(2014)发现烟 水处理显著增加了地中海地区唇形科植物种子的萌 发, 其中Lavandula stoechas的萌发率从 $69 \%$ 增加到 经1:100的烟水处理后的 $95 \%$ 。植物源烟促进了欧洲 温带地区小果亚麻荠(Camelina microcarpa)、荠 (Capsella bursa-pastoris)、播娘蒿 (Descurainia Sophia)和长叶车前(Plantago lanceolata) 4种草本植 物种子的萌发(Mojzes \& Kalapos, 2014)。

相近类群的植物种子萌发对于烟信号的响应并 不完全一致。在同一种群的不同个体水平上, 芸香 科植物Boronia floribunda种子萌发对不同烟水浓度 的响应在个体间存在显著差异, 此外, 相同烟处理 下，个体间萌发开始的时间也存在显著差异( Ma et al., 2018)。在同一物种不同种群水平上, $\mathrm{SP}_{1}$ 和烟水 处理分别显著提高了 Anigozanthos flavidus的 12 和 8 个种群的萌发率(Ma et al., 2020)。

对于不易发生火烧的生态系统中出现的萌发对 于植物源烟的响应现象, Flematti等(2004)认为烟雾 中的生物活性物质是刺激种子萌发的重要原因。 $\mathrm{KARs}$ 和 $\mathrm{SP}_{1}$ 作为烟信号中影响种子萌发的主要生物 活性物质已经被广泛验证(表2)。Daws等(2007)发现 来自不易发生火烧生态系统中的18种杂草的种子经 $\mathrm{KAR}_{1}$ 处理后, 8 种杂草的萌发率显著提高, 这表明 $\mathrm{KAR}_{1}$ 作为萌发刺激物质具有广泛的适应性。但

KARs对种子萌发影响的作用机制尚不明确。KARs 同植物激素独脚金内酯具有结构上的相似性，但是 植物对于二者的响应却不一致(Waters et al., 2014)。 KARs处理使拟南芥(Arabidopsis thaliana)种子的萌 发率显著增加, 科研人员借助该物种丰富的基因组 学资源发现了影响KARs作用机制的两个重要基因 MAX2和KAI2 (Nelson et al., 2012; Waters et al., 2014)。Gupta等(2019)发现烟水和KAR 1 通过降低脱 落酸水平和增强水解酶活性显著促进光敏植物莴苣 (Lactuca sativa) 种子萌发, 并缓解了萌发对于光照 的需求。植物源烟的组成成分复杂, 环荵木科植物 Gyrostemon racemiger种子萌发对烟水响应, 但是对 $\mathrm{KAR}_{1}$ 和 $\mathrm{SP}_{1}$ 不响应, 这表明烟信号中依旧存在一些 新的微量萌发刺激物质尚未确定(Downes et al., 2013)。因此，未来需要进行更多的实验来探讨植物 种子萌发对于火烧信号的响应情况, 特别是要选择 不同生境中的特有物种, 以此来帮助分离和确定烟 信号中影响种子萌发的特定化学物质。

火烧后土壤中的营养物质的组成和有效性会发 生显著变化。氮以铵态氮或有机氮的形式随雨水进 入到土壤中, 随后被硝化细菌转化为硝酸盐。一些 研究表明, 这类含氮化合物(主要是亚硝酸盐和硝 酸盐)能有效地打破许多植物种子的休眠或者促进 其萌发(Baskin \& Baskin, 2014)。例如，硝酸盐显著 
促进了禾本科植物Emmenanthe penduliflora的种子 萌发(Thanos \& Rundel, 1995)。Pérez-Fernández和
Rodríguez-Echeverría (2003)发现, 亚硝酸钠处理显 著提高了鸭茅(Dactylis glomerata)、Cistus ladanifer

表3 物理信号和化学信号交互对种子萌发的影响

Table 3 Effects of interaction of physical and chemical cues on seed germination

\begin{tabular}{lllll}
\hline $\begin{array}{l}\text { 科名 } \\
\text { Family }\end{array}$ & $\begin{array}{l}\text { 物种 } \\
\text { Species }\end{array}$ & $\begin{array}{l}\text { 实验设计 } \\
\text { Experiment design }\end{array}$ & $\begin{array}{l}\text { 响应类型 } \\
\text { Response type }\end{array}$ & $\begin{array}{l}\text { 主要发现 } \\
\text { Main findings }\end{array}$ \\
\hline 豆科 & Harpalyce sp. & $S W(1: 1) \times H S$ & 增效作用 & 萌发率最大值在烟水和热激交互处理下达到 $32 \%$ 。同对照、烟水和热激 \\
Fabaceae & & $\left(100{ }^{\circ} \mathrm{C}-1 \mathrm{~min}\right)$ & Positive effect & 处理相比分别提高了 $19 \%$ 、27\%、25\%(Zirondi et al., 2019)。
\end{tabular}

The maximum germination percentage reached $32 \%$ at the interactive treatment, which was increased by $19 \%, 27 \%$, and $25 \%$ respectively compared with the control, smoke water, and heat shock treatments (Zirondi et al., 2019).

$\begin{array}{llll}\begin{array}{l}\text { 豆科 } \\ \text { Fabaceae }\end{array} & \text { Mimosa leiocephala } & \begin{array}{l}\text { SW }(1: 1) \times H S \\ \left(100{ }^{\circ} \mathrm{C}-1 \mathrm{~min}\right)\end{array} & \begin{array}{l}\text { 增效作用 } \\ \text { Positive effect }\end{array} \\ & & \\ & & \\ \text { 豆科 } & \text { Mimosa somnians } & \begin{array}{l}S W(1: 1) \times H S \\ \left(100{ }^{\circ} \mathrm{C}-1 \mathrm{~min}\right)\end{array} & \begin{array}{l}\text { 增效作用 } \\ \text { Fabaceae }\end{array} \\ & & \end{array}$

萌发率最大值在烟水和热激交互处理下达到 $35 \%$ 。同对照、烟水和热激 处理相比分别提高了 $26 \% 、 21 \% 、 28 \%$ (Zirondi et al., 2019)。

The maximum germination percentage reached $35 \%$ at the interactive treatment, which was increased by $26 \%, 21 \%$, and $28 \%$ respectively compared with the control, smoke water, and heat shock treatments (Zirondi et al., 2019).

萌发率最大值在烟水和热激交互处理下达到 $27 \%$ 。同对照、烟水和热激 处理相比分别提高了 $13 \% 、 6 \% 、 13 \%$ (Zirondi et al., 2019)。

The maximum germination percentage reached $27 \%$ at the interactive treatment, which was increased by $13.0 \%, 6 \%$, and $13.0 \%$ respectively compared with the control, smoke water, and heat shock treatment (Zirondi et al., 2019).

\begin{tabular}{|c|c|c|}
\hline $\begin{array}{l}\text { 豆科 } \\
\text { Fabaceae }\end{array}$ & Acacia angustissima & $\begin{array}{l}S W \times H S \\
\left(120{ }^{\circ} \mathrm{C}-5 \mathrm{~min}\right)\end{array}$ \\
\hline 豆科 & Calliandralo & $S W \times H S$ \\
\hline Fabacea & ngipedicellata & $\left(120{ }^{\circ} \mathrm{C}-5 \mathrm{~min}\right)$ \\
\hline
\end{tabular}

等效作用萌发率在交互处理和热激处理之间无显著差异(Zuloaga-Aguilar et al., Equivalent effect 2011)。

There was no significant difference in germination percentage between the heat shock treatment and the interactive treatment (Zuloaga-Aguilar et al., 2011).

减效作用萌发率在热激处理下达到最大值, 显著高于烟水和热激的交互处理 Negative effect (Zuloaga-Aguilar et al., 2011)。

The germination percentage reached the maximum at the heat shock treatment, which was significantly higher than the interactive treatment (ZuloagaAguilar et al., 2011).

$\begin{array}{llll}\text { 菊科 } & \text { 白紫千里光 } & (\text { Smoke-60 min }) \times H S & \text { 减效作用 } \\ \text { Asteraceae } & \text { Senecio albopurpureus }\left(80{ }^{\circ} \mathrm{C}-5 \mathrm{~min}\right) & \text { Negative effect }\end{array}$

萌发率在热激和烟交互处理下达到最小值, 显著低于热激、烟和对照处 理(Gonzalez \& Ghermandi, 2012)。

The germination percentage reached the minimum at interactive treatment, which was significantly lower than the control, heat shock, and smoke treatments (Gonzalez \& Ghermandi, 2012).

$\begin{array}{lll}\text { 天门冬科 } & \text { Dichopogon strictus } & S W(1: 10) \times H S \\ \text { Asparagaceae } & \left(80^{\circ} \mathrm{C}-1 \mathrm{~h}\right)\end{array}$

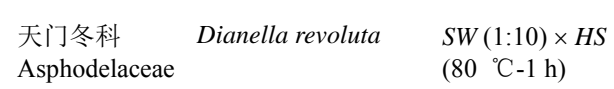

车前科 Penstemon barbatus $S W(1: 10) \times H S$

Plantaginaceae $\quad\left(100{ }^{\circ} \mathrm{C}-30 \mathrm{~min}\right)$

\begin{tabular}{|c|c|c|}
\hline $\begin{array}{l}\text { 翡若翠科 } \\
\text { Velloziaceae }\end{array}$ & Vellozia alutacea & $\begin{array}{l}S W \times H S \\
\left(100{ }^{\circ} \mathrm{C}-5 \mathrm{~min}\right)\end{array}$ \\
\hline $\begin{array}{l}\text { 翡若翠科 } \\
\text { Velloziaceae }\end{array}$ & Vellozia resinosa & $\begin{array}{l}S W \times H S \\
\left(100{ }^{\circ} \mathrm{C}-5 \mathrm{~min}\right)\end{array}$ \\
\hline $\begin{array}{l}\text { 翡若翠科 } \\
\text { Velloziaceae }\end{array}$ & Vellozia epidendroides & $\begin{array}{l}S W \times H S \\
\left(100{ }^{\circ} \mathrm{C}-5 \mathrm{~min}\right)\end{array}$ \\
\hline $\begin{array}{l}\text { 翡若翠科 } \\
\text { Xyridaceae }\end{array}$ & Xyris pilosa & $\begin{array}{l}S W \times H S \\
\left(100{ }^{\circ} \mathrm{C}-5 \mathrm{~min}\right)\end{array}$ \\
\hline
\end{tabular}

增效作用 Positive effect

新鲜种子的萌发率最大值在烟水和热激交互处理下达到 $72 \%$ 。同对照、 烟水、热激处理相比分别提高了 $22 \% 、 19 \% 、 31 \%$ (Hodges et al., 2019)。 The maximum germination percentage reached $72 \%$ at the interactive treatment, which was increased by $22 \%, 19 \%$, and $31 \%$ respectively compared with the control, smoke water, and heat shock treatments (Hodges et al., 2019).

减效作用新鲜种子的萌发率在烟水和热激处理下达到 $33 \%$, 同烟水处理相比下降 Negative effect 了5\% (Hodges et al., 2019)。

The germination percentage reached $33 \%$ at the interactive treatment, which was 5\% lower than the control (Hodges et al., 2019).

等效作用在烟水处理下萌发率最大值达到 $63 \%$, 但与烟水和热激交互处理无显著 Equivalent effect 差异(Abella, 2006)。

The maximum germination percentage reached $63 \%$ at the smoke water treatment, there was no significantly difference with the interactive treatment (Abella, 2006).

减效作用萌发率在烟水和热激交互处理下显著低于烟水处理(Fernandes et al., 2021)。

Negative effect The germination percentage at the interactive treatment was significantly lower than the smoke water treatment (Fernandes et al., 2021).

减效作用萌发率在烟水和热激交互处理下显著低于烟水处理(Fernandes et al., 2021)。 Negative effect The germination percentage at the interactive treatment was significantly lower than the smoke water treatment (Fernandes et al., 2021).

减效作用萌发率在交互处理下显著低于热激处理(Fernandes et al., 2021)。

Negative effect The germination percentage at the interactive treatment was significantly lower than the heat shock treatment (Fernandes et al., 2021).

等效作用萌发率在交互处理和烟水处理之间无显著差异(Fernandes et al., 2021)。

Equivalent effect There was no significant difference in germination percentage between the smoke water treatment and the interactive treatment (Fernandes et al., 2021).

$S W$, 烟水; $H S$, 热激。

SW, smoke water; $H S$, heat shock.

www.plant-ecology.com 
等植物种子的萌发率。

\section{3 物理与化学信号的交互作用}

物理和化学信号共同作用影响种子萌发的现象 普遍存在(表3)。根据萌发率将交互作用的效果分为 增效作用、等效作用、减效作用3种类型。增效作用: 物理和化学信号共同作用下, 萌发率高于单独施加 两种处理。Harpalyce sp.和Mimosa leiocephala在热 激和烟信号交互作用下取得了更高的萌发率，其中 Harpalyce sp. 的萌发率在烟水和热激交互处理下达 到最大值 $32 \%$, 同对照、烟水和热激相比分别提高 了19\%、27\%、25\% (Zirondi et al., 2019)。等效作用: 物理和化学信号共同作用下, 萌发率与施加单独处 理的效果无显著差异。Acacia angustissima在热激单 独处理下与交互处理无显著差异, Penstemon barbatus在烟水单独处理下与交互作用无显著差异 (Abella, 2006; Zuloaga-Aguilar et al., 2011)。减效作 用: 物理和化学信号共同作用下, 萌发率显著低于 单独施加处理的效果。Vellozia alutacea, Vellozia resinosa和白紫千里光(Senecio albopurpureus)等物 种在热激和烟信号交互作用下萌发率受到了显著的 抑制(Gonzalez \& Ghermandi, 2012; Fernandes et al., 2021)。

物理和化学信号交互作用对种子萌发的影响可 能受火烧信号的强度以及种子休眠类型等多种因素 影响。复合休眠的物种在热激打破种子的物理休眠 后, 烟水可以促进种子生理休眠的释放, 起到增效 作用(Auld \& Ooi, 2009)。物理休眠的种子通常会受 到热量的刺激而萌发, 而由于种皮的不透水性, 可 能会导致对烟没有响应以起到等效作用。高浓度的
烟信号和高强度的热激会使种子失去活性，降低种 子萌发率(Ferraz et al., 2013; Liyanage \& Ooi, 2015)。 因此, 当物理和化学信号互作超过了打破种子休眠 所需的阈值就会使种子失去活性或者再次进入休眠 状态, 起到减效作用(Gonzalez \& Ghermandi, 2012)。

\section{4 火烧信号与种子休眠类型之间的关系}

在易发生火烧的生态系统中, 种子的休眠特性 是影响火烧后土壤种子库持久性和幼苗建植的关 键。Baskin和Baskin (2014) 将植物种子的休眠类型划 分为生理休眠、物理休眠、复合休眠、形态休眠和 形态生理休眠。大量研究表明多种休眠类型与火烧 信号存在紧密联系(图3)(Cao et al., 2020; Collette \& Ooi, 2021)。一般情况下, 火烧产生的高温可以打破 物理休眠种子的不透水层, 刺激种子萌发。但是也 有部分物理休眠的植物种子萌发并不受热激效应影 响, 因为不同物种的温度生态幅存在明显差异 (Baskin \& Baskin, 2014)。烟、烟水、火烧灰等化学 信号可以促进种子生理休眠和形态生理休眠的释放, 刺激种子萌发。但是部分具有生理或者形态生理休 眠的种子只有在经过一段时间的后熟处理后，才能 对烟信号产生萌发响应(Flematti et al., 2015)。对于 复合休眠的种子而言热激打破物理休眠后, 化学信 号可以促进种子生理休眠的释放 (Auld \& Ooi, 2009)。特别值得关注的是火烧信号与种子休眠类型 之间并非是严格对应的，例如，Mackenzie等(2016) 发现 $90{ }^{\circ} \mathrm{C}-10 \mathrm{~min}$ 的热激处理显著促进了具有生理 休眠的芸香科植物Boronia serrulata种子的萌发。部 分物理休眠的种子可能会因为烟水呈酸性, 影响其 种皮渗透性而打破休眠(Moreira \& Pausas, 2018)。

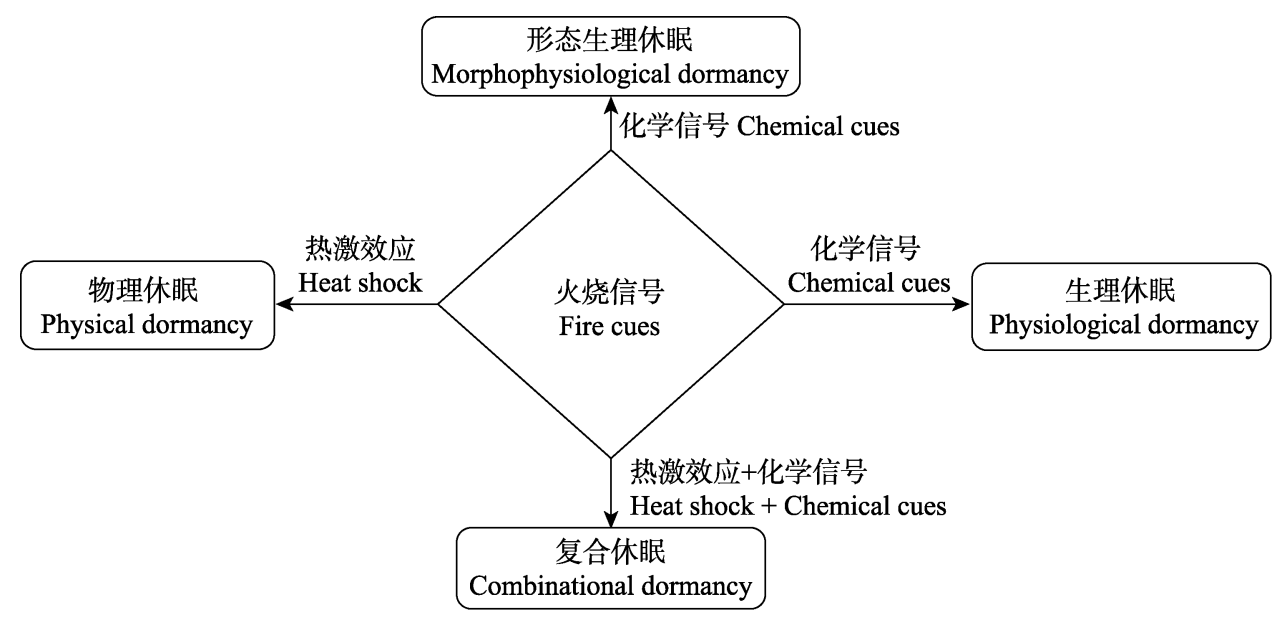

图3 火烧信号与休眠类型之间的关系。

Fig. 3 Relationships between fire cues and dormancy classes. 


\section{3 火烧信号在实践中的应用}

烟信号可以通过提高种子萌发率、促进幼苗生 长发育、增强幼苗活性来增加新生个体的数目和生 物多样性指数以实现退化生态系统的恢复。澳大利 亚西部Banksia林地是火烧易发的地区，其恢复地区 萌发植物的总数增加了 4-48倍, 物种多样性增加了 3倍(Rokich \& Dixon, 2007)。化学信号在农业和园艺 育苗上的应用可以通过对种子或者幼苗施加气态 烟、烟水、KAR 1 等方式实现。烟水对于黄瓜(Cucumis sativus)、番茄(Lycopersicon esculentum)、Gladiolus $\times$ hybridus和金盛花(Calendula officinalis) 4种园艺植 物种子的萌发和幼苗生长具有显著的促进作用 (Elsadek \& Yousef, 2019)。Sparg等(2006)发现烟和烟 水处理显著提高了玉米(Zea mays)的萌发率, 增强 了幼苗活力。将烟水和 $\mathrm{KAR}_{1}$ 直接喷施在幼苗表面可 以促进咖啡黄葵(Abelmoschus esculentus)和番茄幼 苗的生长发育, 具体表现在根长、苗长、叶片的数 量、总叶面积和茎粗的增加(Kulkarni et al., 2011)。 种子萌发和幼苗建植是植物重要的生活史阶段, 也 是对外界环境因子最敏感的时期。将烟或烟水用作 种子引发剂, 为提高种子在盐碱、干旱等逆境条件 下萌发和幼苗建植提供了有效的方法。烟水处理增 加了玉米中 $\mathrm{K}^{+}$和 $\mathrm{Ca}^{2+}$ 的含量, 减少了 $\mathrm{Na}^{+}$的含量, 使 玉米的萌发率从 $70 \%$ 增至 $93 \%$, 根和幼苗的干鲜质 量也有增加(Waheed et al., 2016)。用烟水和KAR $\mathrm{K}_{1}$ 对 芝麻科植物Ceratotheca triloba种子进行引发, 提高 了在极端温度、干旱和盐碱等胁迫环境下种子的萌 发率(Masondo et al., 2018)。但目前植物种子萌发和 幼苗建植的引发技术大部分处于实验研究阶段, 较 少应用于田间实践, 并且缺乏对不同类型引发剂作 用效果的比较和作用机理的深入探讨。

\section{4 总结和展望}

火是陆地生态系统的重要组成部分, 也是影响 许多生态过程的关键因子。本文系统地总结了近些 年来不同类别的火烧信号及其交互作用对种子萌发 影响的研究。为了进一步理解火烧信号对种子萌发 的影响, 我们建议未来的研究可以从以下 4 个方面 入手:

(1)深入探讨烟信号的作用机理。借助模式植物 拟南芥丰富的基因组学资源, 人们对于KARs影响
种子萌发的作用机理有了更深层次的了解。但是拟 南芥并非是易发生火烧生态系统中的典型物种。对 于必须借助火烧信号才能实现萌发的植物来说, 其 对于KARs的响应机制是否同拟南芥一致尚未确定。 KARs作为植物源烟中主要的萌发刺激物质, 探究 其作用机理对于揭示植物生长发育调控新途径具有 重要意义。今后需要通过分子生物学、多组学联合 分析等技术手段，深入探讨烟信号对种子萌发及幼 苗生长的作用机制。

(2)在全球变化的背景下, 探究不断变化的火烧 情势对种子萌发的影响。火烧情势反映了影响特定 生态系统的火烧的大小、频率、扩散速度等特征。 以增温、 $\mathrm{CO}_{2}$ 浓度升高、极端天气频发等为主要特 征的全球变化对火烧事件产生了重要影响。例如, 在过去的半个世纪里, 加拿大森林地区火烧发生的 强度、火烧季节开始和结束的时间均发生了显著的 变化(Hanes et al., 2019)。生物对于火的适应实质是 对不同火烧情势的适应(Keeley et al., 2011)。植物种 子萌发如何响应全球变化背景下火烧情势的改变应 是未来研究的热点和重点。

(3)开展火烧信号同环境因子互作影响种子萌 发的系统研究。水分、氧气、光照、温度等环境因 子决定种子是否萌发。当前, 火烧信号对种子萌发 影响的研究多集中于单一火烧信号或者两种不同类 别的火烧信号的交互实验, 火烧信号与环境因子互 作的探究实验较少。开展火烧信号与环境因子互作 的研究可以更好地反映自然环境中的真实条件, 有 助于更加全面地理解种子萌发对于火烧信号的响应 情况。

(4)加强火烧信号的合理应用。火烧信号作为重 要的生态系统管理工具, 特别是火烧产生的化学信 号, 在农业和园艺育苗、提高种子抗逆性和生态恢 复等方面具有重要的应用潜力。但目前的研究多限 于实验室内小范围的控制实验，如何规模化利用烟 信号为农业和园艺业的发展提供技术支撑还需要进 行深入探讨。

\section{参考文献}

Abella SR (2006). Effects of smoke and fire-related cues on Penstemon barbatus seeds. The American Midland Naturalist, 155, 404-410.

Auld TD, Denham AJ (2006). How much seed remains in the soil after a fire? Plant Ecology, 187, 15-24. 
Auld TD, O'Connell MA (1991). Predicting patterns of postfire germination in 35 eastern Australian Fabaceae. Australian Journal of Ecology, 16, 53-70.

Auld TD, Ooi MKJ (2009). Heat increases germination of water-permeable seeds of obligate-seeding Darwinia species (Myrtaceae). Plant Ecology, 200, 117-127.

Ba LN, Liu Q, Zhang YH, Wang SZ, Gu HY (2018). Effect of smoke, heat shock and ash on seed germination of Populus ussuriensis. Journal of Northeast Forestry University, 46(6), 27-32. [巴丽娜, 刘强, 张芸慧, 王顺忠, 谷会岩 (2018). 烟熏、热激、火烧灰对大青杨种子萌发的影响. 东北林业大学学报, 46(6), 27-32.]

Baeza MJ, Roy J (2008). Germination of an obligate seeder (Ulex parviflorus) and consequences for wildfire management. Forest Ecology and Management, 256, 685-693.

Baskin CC, Baskin JM (2014). Seed: Ecology, Biogeography, and Evolution of Dormancy and Germination. 2nd ed. Academic Press, San Diego, USA.

Baskin JM, Baskin CC, Li X (2000). Taxonomy, anatomy and evolution of physical dormancy in seeds. Plant Species Biology, 15, 139-152.

Bond WJ, Keeley JE (2005). Fire as a global "herbivore": the ecology and evolution of flammable ecosystems. Trends in Ecology \& Evolution, 20, 387-394.

Bond WJ, Woodward FI, Midgley GF (2005). The global distribution of ecosystems in a world without fire. New Phytologist, 165, 525-538.

Bowman DMJS, Balch JK, Artaxo P, Bond WJ, Carlson JM, Cochrane MA, D'Antonio CM, DeFries RS, Doyle JC, Harrison SP, Johnston FH, Keeley JE, Krawchuk MA, Kull CA, Marston JB, et al. (2009). Fire in the earth system. Science, 324, 481-484.

Bradstock RA, Auld TD (1995). Soil temperatures during experimental bushfires in relation to fire intensity: consequences for legume germination and fire management in south-eastern Australia. Journal of Applied Ecology, 32, 76-84.

Brown NAC, van Staden J (1997). Smoke as a germination cue: a review. Plant Growth Regulation, 22, 115-124.

Brys R, Jacquemyn H, de Blust G (2005). Fire increases aboveground biomass, seed production and recruitment success of Molinia caerulea in dry heathland. Acta Oecologica, 28, 299-305.

Cao DC, Baskin CC, Baskin JM (2020). Dormancy class: another fire seasonality effect on plants. Trends in Ecology \& Evolution, 35, 1055-1057.

Çatav ŞS, Küçükakyüz K, Akbaş K, Tavşanoğlu Ç Tavsanoğlu $C$ (2014). Smoke-enhanced seed germination in Mediterranean Lamiaceae. Seed Science Research, 24, 257-264.

Chou YF, Cox RD, Wester DB (2012). Smoke water and heat shock influence germination of shortgrass prairie species. Rangeland Ecology \& Management, 65, 260-267.
Collette JC, Ooi MKJ (2021). Distribution of seed dormancy classes across a fire-prone continent: effects of rainfall seasonality and temperature. Annals of Botany, 127, 613-620.

Coons J, Coutant N, Lawrence B, Finn D, Finn S (2014). An effective system to produce smoke solutions from dried plant tissue for seed germination studies. Applications in Plant Sciences, 2, 1300097. DOI: 10.3732/apps.1300097.

Cross AT, Paniw M, Ojeda F, Turner SR, Dixon KW, Merritt DJ (2017). Defining the role of fire in alleviating seed dormancy in a rare Mediterranean endemic subshrub. $A o B$ Plants, 9, plx036. DOI: 10.1093/aobpla/plx036.

Cuello N, López-Mársico L, Rodríguez C (2020). Field burn versus fire-related cues: germination from the soil seed bank of a South American temperate grassland. Seed Science Research, 30, 206-214.

Daws MI, Davies J, Pritchard HW, Brown NAC, Staden J (2007). Butenolide from plant-derived smoke enhances germination and seedling growth of arable weed species. Plant Growth Regulation, 51, 73-82.

Downes KS, Light ME, Pošta M, Kohout L, van Staden J (2013). Comparison of germination responses of Anigozanthos flavidus (Haemodoraceae), Gyrostemon racemiger and Gyrostemon ramulosus (Gyrostemonaceae) to smoke-water and the smoke-derived compounds karrikinolide (KAR1) and glyceronitrile. Annals of Botany, 111, 489-497.

Downes KS, Light ME, Pošta M, Kohout L, van Staden J (2014). Do fire-related cues, including smoke-water, karrikinolide, glyceronitrile and nitrate, stimulate the germination of 17 Anigozanthos taxa and Blancoa canescens (Haemodoraceae)? Australian Journal of Botany, 62, 347-358.

Elsadek MA, Yousef EA (2019). Smoke-water enhances germination and seedling growth of four horticultural crops. Plants, 8, 104. DOI: 10.3390/plants8040104.

Fernandes AF, Oki Y, Fernandes GW, Moreira B (2021). The effect of fire on seed germination of campo rupestre species in the South American Cerrado. Plant Ecology, 222, 45-55.

Ferraz IDK, Arruda YMBC, van Staden J (2013). Smoke-water effect on the germination of Amazonian tree species. South African Journal of Botany, 87, 122-128.

Flematti GR, Dixon KW, Smith SM (2015). What are karrikins and how were they "discovered" by plants? BMC Biology, $13,1-7$.

Flematti GR, Ghisalberti EL, Dixon KW, Trengove RD (2004). A compound from smoke that promotes seed germination. Science, 305, 977. DOI: 10.1126/science.1099944.

Flematti GR, Merritt DJ, Piggott MJ, Trengove RD, Smith SM, Dixon KW, Ghisalberti EL (2011). Burning vegetation produces cyanohydrins that liberate cyanide and stimulate 
seed germination. Nature Communications, 2, 360. DOI: 10.1038/ncomms 1356 .

Gonzalez SL, Ghermandi L (2012). Fire cue effects on seed germination of six species of northwestern Patagonian grasslands. Natural Hazards and Earth System Sciences, 12, 2753-2758.

Gupta S, Plačková L, Kulkarni MG, Doležal K, van Staden J (2019). Role of smoke stimulatory and inhibitory biomolecules in phytochrome-regulated seed germination of Lactuca sativa. Plant Physiology, 181, 458-470.

Hanes CC, Wang XL, Jain P, Parisien MA, Little JM, Flannigan MD (2019). Fire-regime changes in Canada over the last half century. Canadian Journal of Forest Research, 49, 256-269.

He TH, Lamont BB, Downes KS (2011). Banksia born to burn. New Phytologist, 191, 184-196.

He TH, Lamont BB, Pausas JG (2019). Fire as a key driver of Earth's biodiversity. Biological Reviews, 94, 1983-2010.

Herrero C, Kassa A, Pando V, Bravo F, Alía R (2019). Effect of heat shock on the germination of seeds of the species Acacia senegal L. and Acacia seyal Del. from sub-Saharan Africa (Ethiopia). Forest Systems, 28, e006. DOI: $10.5424 / \mathrm{fs} / 2019282-14227$.

Hodges JA, Price JN, Nimmo DG, Guja LK (2019). Evidence for direct effects of fire-cues on germination of some perennial forbs common in grassy ecosystems. Austral Ecology, 44, 1271-1284.

Kamran M, Khan AL, Waqas M, Imran QM, Hamayun M, Kang SM, Kim YH, Kim MJ, Lee IJ (2014). Effects of plant-derived smoke on the growth dynamics of Barnyard Grass (Echinochloa crusgalli). Acta Agriculturae Scandinavica, Section B-Soil \& Plant Science, 64, 121-128.

Keeley JE, Fotheringham CJ (1998). Smoke-induced seed germination in California chaparral. Ecology, 79, 2320-2336.

Keeley JE, McGinnis TW, Bollens KA (2005). Seed germination of sierra Nevada postfire chaparral species. Madroño, 52, 175-181.

Keeley JE, Pausas JG, Rundel PW, Bond WJ, Bradstock RA (2011). Fire as an evolutionary pressure shaping plant traits. Trends in Plant Science, 16, 406-411.

Kelly LT, Giljohann KM, Duane A, Aquilué N, Archibald S, Batllori E, Bennett AF, Buckland ST, Canelles Q, Clarke MF (2020). Fire and biodiversity in the Anthropocene. Science, 370, eabb0355. DOI: 10.1126/science.abb0355.

Kemp KB, Higuera PE, Morgan P, Abatzoglou JT (2019). Climate will increasingly determine post-fire tree regeneration success in low-elevation forests, Northern Rockies, USA. Ecosphere, 10, e02568. DOI: 10.1002/ecs2.2568.

Kępczyński J, Białecka B, Light ME, Staden J (2006). Regulation of Avena fatua seed germination by smoke solutions, gibberellin A_3 and ethylene. Plant Growth Regulation, 49, 9-16.
Kępczyński J, Cembrowska D, Staden J (2010). Releasing primary dormancy in Avena fatua L. caryopses by smoke-derived butenolide. Plant Growth Regulation, 62, 85-91.

Kulkarni MG, Light ME, van Staden J (2011). Plant-derived smoke: old technology with possibilities for economic applications in agriculture and horticulture. South African Journal of Botany, 77, 972-979.

Liyanage GS, Ooi MKJ (2015). Intra-population level variation in thresholds for physical dormancy-breaking temperature. Annals of Botany, 116, 123-131.

López-Mársico L, Farías-Moreira L, Lezama F, Altesor A, Rodríguez C (2019). Light intensity triggers different germination responses to fire-related cues in temperate grassland species. Folia Geobotanica, 54, 53-63.

Lü AF, Tian HQ (2007). Interaction among climatic change, fire disturbance and ecosystem productivity. Journal of Plant Ecology (Chinese Version), 31, 242-251. [吕爱锋, 田汉勤 (2007). 气候变化、火干扰与生态系统生产力. 植物生态学报, 31, 242-251.]

Lü XT, Reed S, Hou SL, Hu YY, Wei HW, Lü FM, Cui Q, Han XG (2017). Temporal variability of foliar nutrients: responses to nitrogen deposition and prescribed fire in a temperate steppe. Biogeochemistry, 133, 295-305.

Luna B, Moreno JM, Cruz A, Fernández-González F (2007). Heat-shock and seed germination of a group of Mediterranean plant species growing in a burned area: an approach based on plant functional types. Environmental and $E x$ perimental Botany, 60, 324-333.

Ma HY, Erickson TE, Walck JL, Merritt DJ (2020). Interpopulation variation in germination response to fire-related cues and after-ripening in seeds of the evergreen perennial Anigozanthos flavidus (Haemodoraceae). International Journal of Wildland Fire, 29, 950-960.

Ma HY, Wu HT, Ooi MKJ (2018). Within population variation in germination response to smoke cues: convergent recruitment strategies and different dormancy types. Plant and Soil, 427, 281-290.

MacKenzie BDE, Auld TD, Keith DA, Hui FKC, Ooi MKJ (2016). The effect of seasonal ambient temperatures on fire-stimulated germination of species with physiological dormancy: a case study using Boronia (Rutaceae). PLOS ONE, 11, e0156142. DOI: 10.1371/journal.pone.0156142.

MacKenzie DD, Naeth MA (2019). Effect of plant-derived smoke water and potassium nitrate on germination of understory boreal forest plants. Canadian Journal of Forest Research, 49, 1540-1547.

Masondo NA, Kulkarni MG, Finnie JF, van Staden J (2018). Influence of biostimulants-seed-priming on Ceratotheca triloba germination and seedling growth under low temperatures, low osmotic potential and salinity stress. ECOtoxicology and Environmental Safety, 147, 43-48. 
McLauchlan KK, Higuera PE, Miesel J, Rogers BM, Schweitzer J, Shuman JK, Tepley AJ, Varner JM, Veblen TT, Adalsteinsson SA, Balch JK, Baker P, Batllori E, Bigio E, Brando P, et al. (2020). Fire as a fundamental ecological process: research advances and frontiers. Journal of Ecology, 108, 2047-2069.

Milberg P, Andersson L, Thompson K (2000). Large-seeded species are less dependent on light for germination than small-seeded ones. Seed Science Research, 10, 99-104.

Mojzes A, Kalapos T (2014). Plant-derived smoke stimulates germination of four herbaceous species common in temperate regions of Europe. Plant Ecology, 215, 411-415.

Moreira B, Pausas JG (2018). Shedding light through the smoke on the germination of Mediterranean Basin flora. South African Journal of Botany, 115, 244-250.

Moreira B, Tormo J, Estrelles E, Pausas JG (2010). Disentangling the role of heat and smoke as germination cues in Mediterranean Basin flora. Annals of Botany, 105, 627-635.

Morgan P, Hardy CC, Swetnam TW, Rollins MG, Long DG (2001). Mapping fire regimes across time and space: understanding coarse and fine-scale fire patterns. International Journal of Wildland Fire, 10, 329-342.

Nelson DC, Flematti GR, Ghisalberti EL, Dixon KW, Smith SM (2012). Regulation of seed germination and seedling growth by chemical signals from burning vegetation. Annual Review of Plant Biology, 63, 107-130.

Ooi MKJ, Denham AJ, Santana VM, Auld TD (2014). Temperature thresholds of physically dormant seeds and plant functional response to fire: variation among species and relative impact of climate change. Ecology and Evolution, 4, 656-671.

Pausas JG, Keeley JE (2019). Wildfires as an ecosystem service. Frontiers in Ecology and the Environment, 17, 289-295.

Pérez-Fernández MA, Rodríguez-Echeverría S (2003). Effect of smoke, charred wood, and nitrogenous compounds on seed germination of ten species from woodland in centralwestern Spain. Journal of Chemical Ecology, 29, 237-251.

Preston CA, Baldwin IT (1999). Positive and negative signals regulate germination in the post-fire annual, Nicotiana attenuata. Ecology, 80, 481-494.

Purdie RW (1977). Early stages of regeneration after burning in dry sclerophyll vegetation. II. Regeneration by seed germination. Australian Journal of Botany, 25, 35.

Qiu J, Bai YG, Fu YB, Wilmshurst JF (2010). Spatial variation in temperature thresholds during seed germination of remnant Festuca hallii populations across the Canadian prairie. Environmental and Experimental Botany, 67, 479-486.

Quintana JR, Cruz A, Fernández-González F, Moreno JM (2004). Time of germination and establishment success after fire of three obligate seeders in a Mediterranean shrubland of central Spain. Journal of Biogeography, 31, 241-249.

Ramos DM, Valls JFM, Borghetti F, Ooi MKJ (2019). Fire cues trigger germination and stimulate seedling growth of grass species from Brazilian savannas. American Journal of Botany, 106, 1190-1201.

Reyes O, Trabaud L (2009). Germination behaviour of 14 Mediterranean species in relation to fire factors: smoke and heat. Plant Ecology, 202, 113-121.

Ribeiro LC, Pedrosa M, Borghetti F (2013). Heat shock effects on seed germination of five Brazilian savanna species. Plant Biology, 15, 152-157.

Rivas M, Reyes O, Casal M (2006). Influence of heat and smoke treatments on the germination of six leguminous shrubby species. International Journal of Wildland Fire, $15,73-80$.

Rodríguez-Trejo DA, Fulé PZ (2003). Fire ecology of Mexican pines and a fire management proposal. International Journal of Wildland Fire, 12, 23-37.

Roeder M, Yang WD, Tomlinson KW (2019). Influence of smoke, heat and fire on germination of woody species occurring in the dry valleys of southwest China. Journal of Plant Ecology, 12, 931-940.

Rokich DP, Dixon KW (2007). Recent advances in restoration ecology, with a focus on the Banksia woodland and the smoke germination tool. Australian Journal of Botany, 55, 375-389.

Scaffidi A, Waters MT, Skelton BW, Bond CS, Sobolev AN, Bythell-Douglas R, McKinley AJ, Dixon KW, Ghisalberti EL, Smith SM, Flematti GR (2012). Solar irradiation of the seed germination stimulant karrikinolide produces two novel head-to-head cage dimers. Organic \& Biomolecular Chemistry, 10, 4069-4073.

Shikwambana L, Kganyago M (2021). Observations of emissions and the influence of meteorological conditions during wildfires: a case study in the USA, Brazil, and Australia during the 2018/19 period. Atmosphere, 12, 11. DOI: 10.3390/atmos12010011.

Silveira MVF, Petri CA, Broggio IS, Chagas GO, Macul MS, Leite CCSS, Ferrari EMM, Amim CGV, Freitas ALR, Motta AZV, Carvalho LME, Silva CHL Jr, Anderson LO, Aragão LEOC (2020). Drivers of fire anomalies in the Brazilian Amazon: lessons learned from the 2019 fire crisis. Land, 9, 516. DOI: 10.3390/land9120516.

Sparg SG, Kulkarni MG, van Staden J (2006). Aerosol smoke and smoke-water stimulation of seedling vigor of a commercial maize cultivar. Crop Science, 46, 1336-1340.

Staden JV, Brown NAC, Jäger AK, Johnson TA (2000). Smoke as a germination cue. Plant Species Biology, 15, 167-178.

Stevens JC, Merritt DJ, Flematti GR, Ghisalberti EL, Dixon KW (2007). Seed germination of agricultural weeds is promoted by the butenolide 3-methyl-2H-furo[2,3-c] 
pyran-2-one under laboratory and field conditions. Plant and Soil, 298, 113-124.

Stevens-Rumann CS, Morgan P (2019). Tree regeneration following wildfires in the western US: a review. Fire Ecology, 15, 1-17.

Tavşanoğlu Ç (2011). Fire-related cues (heat shock and smoke) and seed germination in a Cistus creticus population in southwestern Turkey. Ekoloji, 20, 99-104.

Tavşanoğlu Ç, Ergan G, Çatav ŞS, Zare G, Küçükakyüz K, Özüdoğru B (2017). Multiple fire-related cues stimulate germination in Chaenorhinum rubrifolium (Plantaginaceae), a rare annual in the Mediterranean Basin. Seed Science Research, 27, 26-38.

Thanos CA, Rundel PW (1995). Fire-followers in chaparral: nitrogenous compounds trigger seed germination. Journal of Ecology, 83, 207-216.

Todorović S, Božić D, Simonović A, Filipović B, Dragićević M, Giba Z, Grubišić D (2010). Interaction of fire-related cues in seed germination of the potentially invasive species Paulownia tomentosa Steud. Plant Species Biology, 25, 193-202.

van de Venter HA, Esterhuizen AD (1988). The effect of factors associated with fire on seed germination of Erica sessiliflora and E. hebecalyx (Ericaceae). South African Journal of Botany, 54, 301-304.

Veenendaal EM, Torello-Raventos M, Miranda HS, Sato NM, Oliveras I, van Langevelde F, Asner GP, Lloyd J (2018). On the relationship between fire regime and vegetation structure in the tropics. New Phytologist, 218, 153-166.

Visscher AM, Yeo M, Gomez Barreiro P, Stuppy W, Latorre Frances A, Di Sacco A, Seal CE, Pritchard HW (2018). Dry heat exposure increases hydrogen peroxide levels and breaks physiological seed coat-imposed dormancy in $\mathrm{Me}$ sembryanthemum crystallinum (Aizoaceae) seeds. Environmental and Experimental Botany, 155, 272-280.

Waheed M, Jamil M, Khan MD, Shakir SK, Rehman S (2016). Effect of plant-derived smoke solutions on physiological and biochemical attributes of maize (Zea mays L.) under salt stress. Pakistan Journal of Botany, 48, 1763-1774.

Wang X, Xiang CH, Li XW, Wen DJ (2013). Effects of a winter wildfire on plant community structure and forage quality in subalpine grassland of western Sichuan, China. Chinese Journal of Plant Ecology, 37, 922-932. [王谢, 向成 华, 李贤伟, 文冬菊 (2013). 冬季火对川西亚高山草地 植物群落结构和牧草质量的影响. 植物生态学报, 37 , 922-932.]

Waters MT, Scaffidi A, Sun YK, Flematti GR, Smith SM (2014). The karrikin response system of Arabidopsis. The Plant Journal, 79, 623-631.

Yang J, Kong JJ, Liu B (2013). A review of effects of fire disturbance on understory vegetation in boreal coniferous forest. Chinese Journal of Plant Ecology, 37, 474-480. [杨 健, 孔健健, 刘波 (2013). 林火干扰对北方针叶林林下 植被的影响. 植物生态学报, 37, 474-480.]

Yao J, Waters MT (2020). Perception of karrikins by plants: a continuing Enigma. Journal of Experimental Botany, 71, 1774-1781.

Yue C, Luo CF, Shu LF, Shen ZH (2020). Review on wildfire studies in the context of global change. Acta Ecologica Sinica, 40, 385-401. [岳超, 罗彩访, 舒立福, 沈泽吴 (2020). 全球变化背景下的野火研究进展. 生态学报, 40, 385-401.]

Zhou JF, Teixeira da Silva JA, Ma GH (2014). Effects of smoke water and karrikin on seed germination of 13 species growing in China. Central European Journal of Biology, 9, 1108-1116.

Zirondi HL, Silveira FAO, Fidelis A (2019). Fire effects on seed germination: heat shock and smoke on permeable vs impermeable seed coats. Flora, 253, 98-106.

Zuloaga-Aguilar S, Briones O, Orozco-Segovia A (2011). Seed germination of montane forest species in response to ash, smoke and heat shock in Mexico. Acta Oecologica, 37, 256-262. 